Article

\title{
Structural Analyses of Helicobacter Pylori FolC Conducting Glutamation in Folate Metabolism
}

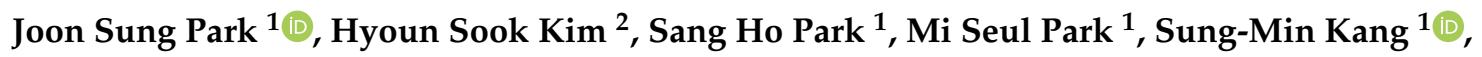 \\ Hyun-Jung Kim ${ }^{3}$ and Byung Woo Han ${ }^{1, *(D)}$ \\ 1 Research Institute of Pharmaceutical Sciences, College of Pharmacy, Seoul National University, 1 Gwanak-ro, \\ Gwanak-gu, Seoul 08826, Korea \\ 2 Division of Precision Medicine, National Cancer Center, 323 Ilsan-ro, Ilsandong-gu, Goyang-si, \\ Gyeonggi-do 10408, Korea \\ 3 College of Pharmacy, Chung-Ang University, 84 Heukseok-ro, Dongjak-gu, Seoul 06974, Korea \\ * Correspondence: bwhan@snu.ac.kr; Tel.: +82-2-880-7898
}

Received: 26 July 2019; Accepted: 17 August 2019; Published: 19 August 2019

check for

\begin{abstract}
FolC plays important roles in the folate metabolism of cells by attaching L-Glu to dihydropteroate (DHP) and folate, which are known activities of dihydrofolate synthetase (DHFS) and folylpolyglutamate synthetase (FPGS), respectively. Here, we determined the crystal structure of Helicobacter pylori FolC $(\mathrm{HpFolC})$ at $1.95 \AA$ resolution using the single-wavelength anomalous diffraction method. HpFolC has globular N- and C-terminal domains connected by a single loop, and a binding site for ATP is located between the two domains. Apo-HpFolC was crystallized in the presence of citrate in a crystallization solution, which was held in the ATP-binding site. Structural motifs such as the P-loop and $\Omega$-loop of $H p$ FolC for binding of ATP and two magnesium ions are well conserved in spite of the low overall sequence similarity to other FolC/FPGSs. The $\Omega$-loop would also recognize a folate molecule, and the DHP-binding loop of $H p F o l C$ is expected to exhibit a unique recognition mode on DHP, compared with other FolCs. Because human FolC is known to only exhibit FPGS activity, the DHFS activity of bacterial FolC is an attractive target for the eradication of pathogenic bacteria. Consequently, our structural analyses of $\mathrm{HpFolC}$ provide a valuable foundation for a universal antibacterial strategy against $H$. pylori as well as other pathogenic bacteria.
\end{abstract}

Keywords: bifunctional FolC; dihydrofolate synthetase (DHFS); folate metabolism; folylpolyglutamate synthetase (FPGS); Helicobacter pylori

\section{Introduction}

Helicobacter pylori is a pathogenic Gram-negative bacterium, which has been classified as a class 1 carcinogen that contributes to chronic inflammation within the human gastric niche and can lead to stomach cancer [1-3]. Eradication of $H$. pylori usually starts with triple therapy, which is a combination of a proton pump inhibitor, amoxicillin, and clarithromycin. Failure of this therapy as a result of antibiotic resistance to clarithromycin has been combated with newly developed approaches, including bismuth and tetracycline, but long-term plans still require novel antibacterial targets for $H$. pylori $[4,5]$.

In cells, folate plays a central role in the single-carbon transfer system involved in DNA (thymidine and purines) and amino acid (Gly, Ser, and Met) synthesis; hence, it is essential for cell growth and replication [6]. Folate and its derivatives in cells are reported to exist mainly in polyglutamated forms, which were initially speculated to be a reservoir of cellular glutamate [6] and, further, to be substrate forms with elevated affinities to several folate-processing enzymes [7-9]. Several folate-derived drugs targeting folate metabolism also exhibit increased efficiencies by polyglutamation [10]. Folate polyglutamation is achieved through folylpolyglutamate synthetase (FPGS, EC 6.3.2.17), which attaches 
L-Glu to acid moieties of folate and its polyglutamated derivatives in an ATP-dependent manner (Figure 1).

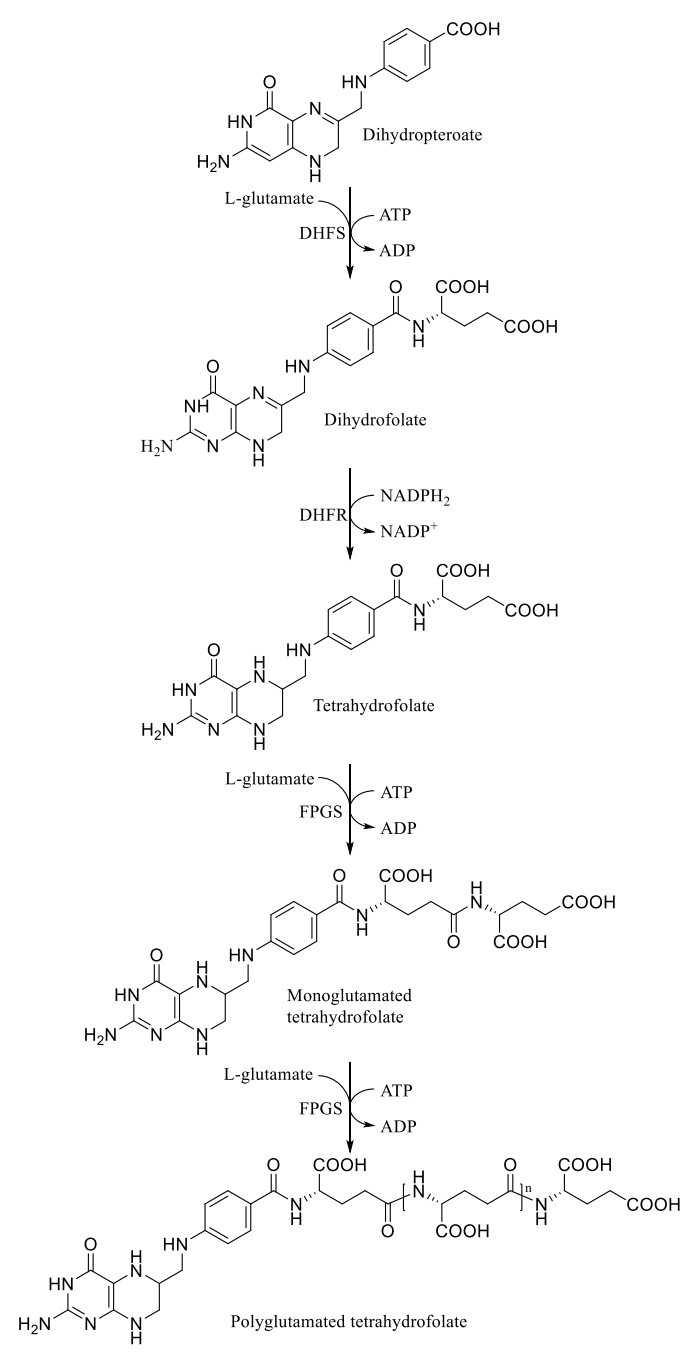

Figure 1. Activities of dihydrofolate synthetase (DHFS) and folylpolyglutamate synthetase (FPGS) in folate metabolism.

FPGS is known as a ubiquitous enzyme found in all living cells. Interestingly, in some organisms such as Corynebacterium species, Escherichia coli, Neisseria gonorrhoeae, Mycobacterium tuberculosis, and Plasmodium falciparum, FPGS has been reported to additionally perform the activity of dihydrofolate synthetase (DHFS, EC 6.3.2.12) as a bifunctional enzyme (referred to as FolC in this paper) and is involved in the de novo synthesis of folate [11-15] (Figure 1). H. pylori is also predicted to have bifunctional FPGS exhibiting DHFS activity [16], which implies that a selective inhibitor against the DHFS activity of $H$. pylori FolC $(\mathrm{HpFolC})$ could be an attractive candidate for an antibacterial drug [17].

So far, structures of FPGSs from five different bacterial species have been determined: E. coli FolC (EcFolC, PDB codes: 1W78 and 1W7K) [18], Lactobacillus casei FPGS (LcFPGS, PDB codes: 1FGS, 1JBV, 1JBW, 2GC5, 2GC6, 2GCA, and 2GCB) [19-21], M. tuberculosis FolC (MtFolC, PDB codes: 2VOR and 2VOS) [22], Thermotoga maritima FolC (TmFolC, PDB code: 1O5Z), and Yersinia pestis FolC (YpFolC, PDB codes: 3N2A, 3NRS, 3PYZ, and 3QCZ). Here, we determined the crystal structure of $H p F o l C$ at $1.95 \AA$ resolution using the single-wavelength anomalous diffraction (SAD) method. We analyzed the structure of $\mathrm{HpFolC}$ in comparison with the other FolC/FPGS structures, which should enable a deeper understanding of folate polyglutamation in $\mathrm{H}$. pylori and provide a structural basis for the development of new antibiotics against $H$. pylori. 


\section{Materials and Methods}

\subsection{Cloning, Protein Expression, and Purification}

The HpFolC gene ( $h p \_1545$, residues 1-394) was cloned into the pET-21a(+) vector (Novagen, Darmstadt, Germany) with a C-terminal hexahistidine tag. The recombinant vector was transformed into Rosetta 2(DE3), an E. coli strain. The transformed cells were selected and cultured with Luria-Bertani media containing $50 \mu \mathrm{g} / \mathrm{mL}$ ampicillin. Cells were shake-incubated at $37^{\circ} \mathrm{C}$ until $\mathrm{OD}_{600}$ reached 0.5 , and $0.5 \mathrm{mM}$ isopropyl $\beta$-D-1-thiogalactopyranoside (IPTG) was added for induction. After induction, the cells were incubated for an additional $16 \mathrm{~h}$ at $20^{\circ} \mathrm{C}$. Cultured cells were harvested at $6,000 \times g$ and lysed by sonication in a buffer containing $20 \mathrm{mM}$ Tris- $\mathrm{HCl}(\mathrm{pH} 7.5), 500 \mathrm{mM} \mathrm{NaCl}, 35 \mathrm{mM}$ imidazole, and $1 \mathrm{mM}$ phenylmethanesulfonyl fluoride. The cell lysate was centrifuged at 35,000× $g$ for $1 \mathrm{~h}$ and the supernatant was filtered with $0.45 \mu \mathrm{m}$ syringe filter (Sartorius, Göttingen, Germany). The filtrate was loaded onto a HiTrap chelating HP column (GE Healthcare, Chicago, IL, USA) and eluted by adding an increasing gradient of a buffer containing $20 \mathrm{mM}$ Tris- $\mathrm{HCl}(\mathrm{pH} 7.5), 500 \mathrm{mM} \mathrm{NaCl}$, and 1 M imidazole. The eluted fractions containing $\mathrm{HpFolC}$ protein were desalted using a HiPrep 26/10 desalting column (GE Healthcare, Chicago, IL, USA) with a buffer containing $20 \mathrm{mM}$ Tris- $\mathrm{HCl}$ (pH 9.0) and $50 \mathrm{mM} \mathrm{NaCl}$. The desalted sample was loaded onto a HiTrap $5 \mathrm{ml}$ Q HP column (GE Healthcare, Chicago, IL, USA) and eluted by adding an increasing gradient of a buffer containing $20 \mathrm{mM}$ Tris- $\mathrm{HCl}(\mathrm{pH} 9.0)$ and $1 \mathrm{M}$ $\mathrm{NaCl}$. The eluted protein was loaded onto a HiLoad 16/600 Superdex 200 pg column (GE Healthcare, Chicago, IL, USA) equilibrated with a buffer containing $10 \mathrm{mM}$ HEPES-NaOH (pH 7.0) and $100 \mathrm{mM}$ $\mathrm{NaCl}$. The eluted protein was concentrated for crystallization.

Selenomethionine (SeMet)-derived HpFolC was expressed with B834(DE3), a methionine auxotrophic E. coli strain. The transformed cells were cultured in the media containing M9 minimal salts (Sigma-Aldrich, Darmstadt, Germany) and an amino acid mix with L-selenomethionine. Cells were shake-incubated at $37^{\circ} \mathrm{C}$ until $\mathrm{OD}_{600}$ reached 0.5 , and $0.5 \mathrm{mM}$ IPTG was added for induction. After induction, the cells were incubated for an additional $16 \mathrm{~h}$ at $20{ }^{\circ} \mathrm{C}$. The subsequent purification procedure was the same as for native $H p$ FolC.

\subsection{Crystallography}

Initial crystal of $\mathrm{HpFolC}$ was obtained using the sitting-drop vapor diffusion method from a commercial crystallization-screening reagent containing 20\% (w/v) polyethylene glycol 3350 and $0.2 \mathrm{M}$ ammonium citrate tribasic (pH 7.0) (The Classic II suite; QIAGEN, Hilden, Germany). HpFolC crystals were further optimized using the hanging-drop vapor diffusion method for $X$-ray diffraction experiments. Before exposure to X-ray beams, crystals were soaked in the crystallization solution supplemented with $25 \%$ glycerol for cryoprotection and flash-cooled in a $100 \mathrm{~K}$ nitrogen gas stream. Diffraction data were indexed and scaled with HKL2000 [23]. The phase of the SeMet-derived crystal was solved using the SAD method with the Autosol program [24,25], and the obtained initial model was used as the template for MR phasing of native crystal data with the Phaser program [26]. Models were built, refined, and validated with Coot [27], phenix.refine [28], and MolProbity [29], respectively. Statistics for data collection and model refinement are summarized in Table 1.

Table 1. Statistics for data collection and model refinement.

\begin{tabular}{ccc}
\hline & H $p$ FolC & SeMet-substituted $H p$ FolC \\
\hline PDB code & 6 K8C & \\
Deata collection & & \\
Space group & & PLS-5C \\
Wavelength $(\AA)$ & $C 2$ & $P 4_{1} 21_{1} 2$ \\
Unit cell parameter & 0.9795 & 0.9798 (Peak) \\
$a, b, c(\AA)$ & $131.45,61.64,69.74$ & $117.90,117.90,69.18$ \\
\hline
\end{tabular}


Table 1. Cont.

\begin{tabular}{|c|c|c|}
\hline & HpFolC & SeMet-substituted $\mathrm{H} p$ FolC \\
\hline$\alpha, \beta, \gamma\left({ }^{\circ}\right)$ & $90,115.96,90$ & $90,90,90$ \\
\hline Resolution range $(\AA)$ & $50.00-1.95(1.98-1.95)^{1}$ & $50.00-2.50(2.54-2.50)^{1}$ \\
\hline No. of unique reflections & $36178(3591)^{1}$ & $17401(1701)^{1}$ \\
\hline$I / \sigma(I)$ & $26.5(4.2)^{1}$ & $69.2(15.9)^{1}$ \\
\hline Wilson B-factor $\left(\AA^{2}\right)$ & 33.9 & 28.4 \\
\hline$R_{\text {merge }}(\%)$ & $0.059(0.431)^{1}$ & $0.148(0.635)^{1}$ \\
\hline Redundancy & $7.4(7.5)^{1}$ & $38.1(39.9)^{1}$ \\
\hline \multirow[t]{2}{*}{ Completeness (\%) } & $98.9(98.9)^{1}$ & $100.0(100.0)^{1}$ \\
\hline & Refinement & \\
\hline Resolution & $30.00-1.95(2.02-1.95)^{1}$ & \\
\hline$R_{\text {work }} / R_{\text {free }}{ }^{2}$ & $0.184 / 0.219$ & \\
\hline \multicolumn{3}{|l|}{ R.m.s. deviations } \\
\hline Bonds $(\AA)$ & 0.004 & \\
\hline Angles $\left(^{\circ}\right)$ & 0.640 & \\
\hline No. of non-H atoms & 3362 & \\
\hline Protein & 3029 & \\
\hline Ligand & $19^{3}$ & \\
\hline Water & 314 & \\
\hline Average B-factors $\left(\AA^{2}\right)$ & 41.22 & \\
\hline Protein & 40.42 & \\
\hline Ligand & 62.68 & \\
\hline Water & 47.59 & \\
\hline Ramachandran favored/outlier (\%) & $98.4 / 0.0^{4}$ & \\
\hline Rotamer outlier $(\%)$ & 0.00 & \\
\hline
\end{tabular}

\subsection{Data Availibility}

Atomic coordinates and structure factor files of the $\mathrm{HpFolC}$ structure described in this research have been deposited in the Protein Data Bank (www.wwpdb.org) with the accession code 6K8C.

\section{Results and Discussions}

\subsection{HpFolC Shares Structural Features of Other FolC/FPGSs}

$\mathrm{HpFolC}$ largely contains N- and C-terminal domains separated by a turn (Ile272 and Gly273) (Figure 2a,b). The $\mathrm{N}$-terminal domain (residues 1-272) contains a mixed $\beta$-sheet of six parallel strands and one antiparallel strand flanked by 13 helices (10 $\alpha$-helices and $33_{10}$-helices), and the C-terminal domain (residues 273-394) contains a mixed $\beta$-sheet of five parallel strands and one antiparallel strand flanked by four helices ( $3 \alpha$-helices and $1 \alpha-3_{10}$ mixed helix, $\left.\alpha \eta 15\right)$. The $\mathrm{N}$ - and C-terminal domains form an interface with a hollow cavity inside, and a citrate molecule from the crystallization solution seems to be captured (Figure 2c). FolC is classified as a member of the Mur ligase family, in which other member proteins are usually involved in peptidoglycan synthesis as amino acid ligases. The overall structure of $\mathrm{H} p \mathrm{FolC}$ is quite similar to other FolC/FPGS structures from Dali analyses (Z-score: $31.8-36.1$ ). An outstanding topological difference of $\mathrm{HpFolC}$ is its $\alpha 12$ in the N-terminal domain, where the other reported FolC/FPGS structures have an antiparallel $\beta$-sheet with three strands instead (Figure 2b).

$\mathrm{H} p \mathrm{FolC}$ was observed to share characteristic functional components, such as the dihydropteroate (DHP)-binding loop ( $\alpha 1-\alpha 2$, residues 18-27), P-loop (phosphate-binding loop, $\beta 1-\alpha 3$, residues $54-58$ ), and $\Omega$-loop ( $\beta 2-\beta 3$, residues $80-90$ ) (Figure 2a). Considering two activities of FolC, dihydropteroate, tetrahydrofolate, ATP, and L-Glu could be the substrates of $\mathrm{HpFolC}$. Among the 16 reported FolC/FPGS structures from five species, two structures (PDB codes: 1W78 and 1JBW) were elucidated in complex with phosphorylated dihydropteroate (DHP-P) [18] and 5,10-methylene-6-hydrofolate [20], respectively, and nine structures (PDB codes: 1JBW, 1JBV, 1W7K, 1W78, 3QCZ, 3PYZ, 3N2A, 2VOR, and 2VOS) 
were elucidated in complex with ATP or its homologues $[18,20,22]$. The $Y p$ FolC structure (PDB code: 3QCZ) was elucidated in complex with the substrate glutamate.

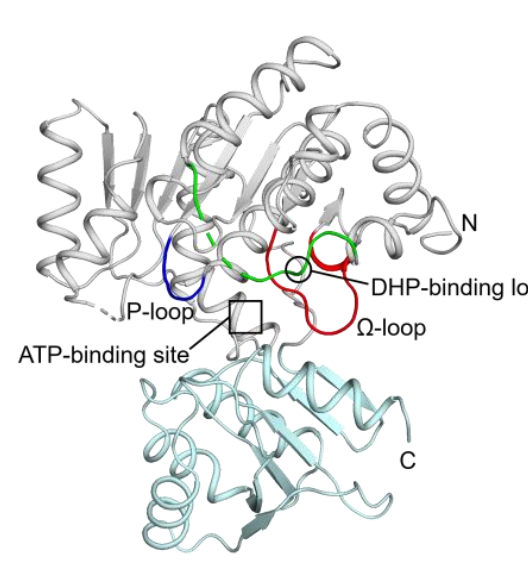

(a)

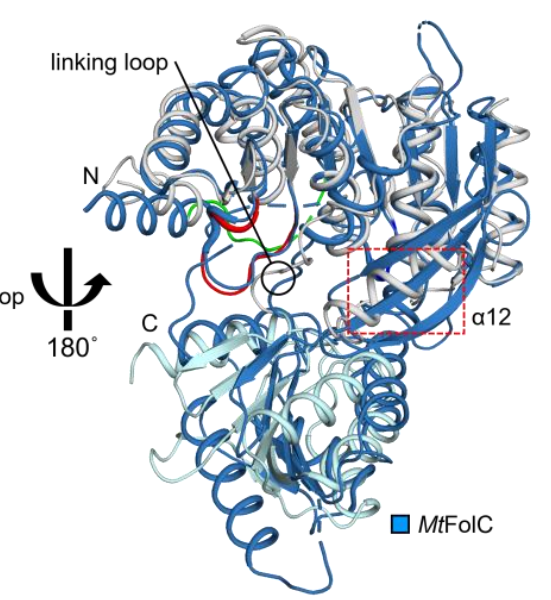

(b)

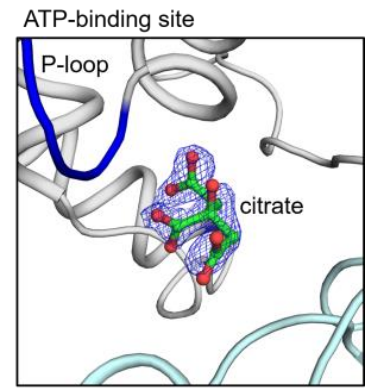

(c)

Figure 2. The overall structure of $H p F o l C$. (a) The crystal structure of $H p$ FolC is shown in a cartoon representation. The $\mathrm{N}$ - and $\mathrm{C}$-terminal domains are colored in white and pale cyan, respectively. The dihydropteroate (DHP)-binding loop, P-loop, and $\Omega$-loop are colored in green, blue, and red, respectively. The ATP-binding site is marked with a black solid square. (b) The structure of $\mathrm{HpFolC}$ from (a) is rotated horizontally by $180^{\circ}$. In addition, the crystal structure of Mycobacterium tuberculosis FolC (MtFolC) (PDB code: 2VOR) is superposed and colored in blue. The loops linking the $\mathrm{N}$ - and C-terminal domains in $\mathrm{HpFolC}$ and $M t$ FolC are marked with a black solid circle, and the helix $\alpha 12$ region of $\mathrm{HpFolC}$ is marked with a red square. (c) The ATP-binding site of HpFolC shown in (a) is magnified. The bound citrate in the crystal structure of $H p F o l C$ is shown with green sticks, and its $2 m F o-D F c$ electron density map is shown with blue meshes at a $1.5 \sigma$ contour level. Oxygen atoms are colored in red.

\subsection{Binding Site of HpFolC for DHP in the Vicinity of the DHP-Binding Loop}

The DHP-binding loop of $\mathrm{HpFolC}$ is mainly composed of charged/aromatic residues $\left({ }^{18}\right.$ TKPKEYHKFD $\left.{ }^{27}\right)$. Previous studies on EcFolC revealed that hydrophobic residues on the DHP-binding loop (Ile28 and Leu30) form a stacking interaction with DHP-P together with Phe124 and Ala155 [18]. The stacking interaction causes the DHP-binding loop of EcFolC to become ordered and to have the "closed" conformation in complex with DHP. Additionally, adjacent polar residues (Thr122, Glu125, and Asp154) of EcFolC interact with hydrogen-bond donors/acceptors of the pterin moiety. Considering the bifunctional properties of FolC, the DHP-binding site would be conserved among FolCs. Our sequence alignment with $\mathrm{HpFolC}, \mathrm{MtFolC}, E c F o l C$, and $L c F P G S$ showed that the corresponding residues to Thr122, Phe124, Glu125, and Asp154 of EcFolC are well-conserved in the other proteins (Figure 3). These conserved residues are thought to interact with DHP as in EcFolC. However, the sequence of the DHP-binding loop of $E c F o l C$ is not conserved. Instead of the hydrophobic stacking of $E c F$ FolC with DHP, the charged/aromatic residues of the DHP-binding loop in HpFolC could form probable hydrogen-bond/hydrophobic/stacking interactions with the pterin moiety of DHP. 


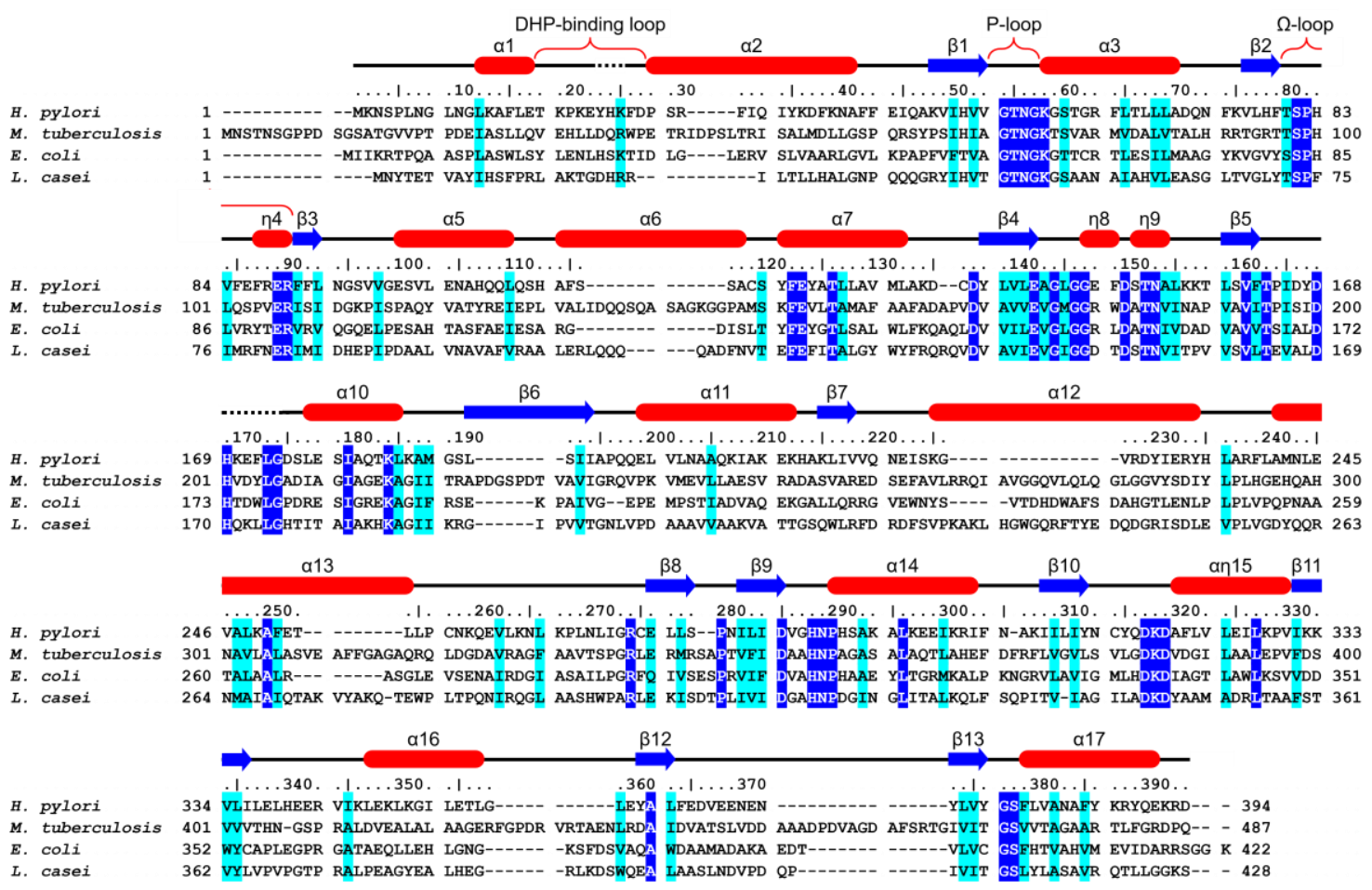

Figure 3. Sequence alignment of $H p F o l C$ with structure-determined FolC/FPGSs. The FolC/FPGS sequences are from H. pylori, M. tuberculosis, Escherichia coli, and Lactobacillus casei. Identical and highly conserved residues are colored in blue and cyan, respectively. Secondary structures of $H p$ FolC are shown above its sequence.

\subsection{P-Loop, a Structural Motif of HpFolC for ATP Binding}

$\mathrm{H} p$ FolC features the P-loop (residues 54-60, GTNGKGS), which is conserved among other FolC/FPGSs as the glycine-rich sequence "GTNGKx(S/T)" (Figure 3). Previous studies on complex structures of FolC/FPGSs with ATP-homologue molecules revealed that an ATP is sandwiched between the $\mathrm{N}$ - and C-terminal domains of FolC/FPGS. Especially, the P-loop provides contacts and interactions with ATP from the N-terminal domain of FolC/FPGS [18,20,22]: the Asn residue forms a hydrogen bond with the ribose moiety of ATP, the "GKx" sequence interacts with the $\beta$-phosphate of ATP, and the " $x(\mathrm{~S} / \mathrm{T})$ " sequence interacts with the $\alpha$-phosphate with its backbone and the side chain of the " $(\mathrm{S} / \mathrm{T})$ " residue. Based on similarities of sequence and structure, the P-loop of $\mathrm{H} p$ FolC seems to recognize an ATP in a similar way (Figure 4 b).

At the opposite side of the P-loop, binding of the ribose moiety, $\alpha$-phosphate, and $\gamma$-phosphate would be stabilized by hydrogen bonds with strictly conserved Asp285, Arg274, and His288 of HpFolC, respectively, as in LcFPGS (Figure 4c). The side chain of His 288 of $\mathrm{HpFolC}$ in our structure is out of binding conformation and expected to move toward the ATP-binding site with ATP binding. 


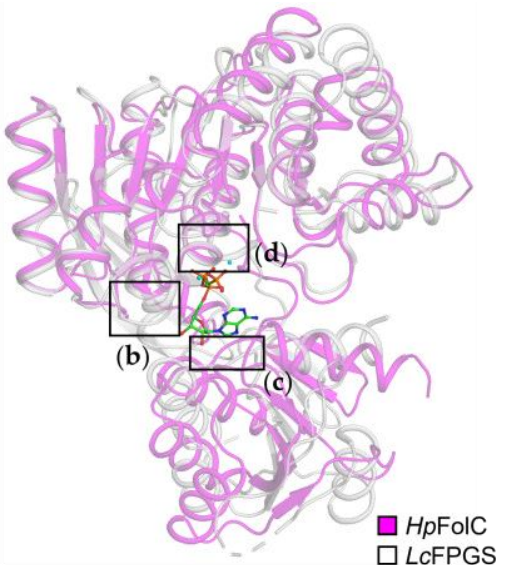

(a)

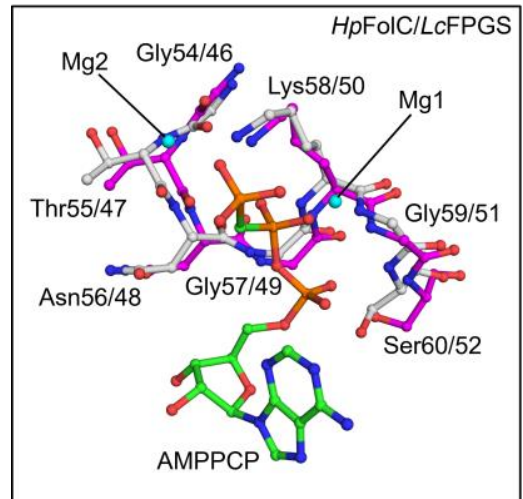

(b)

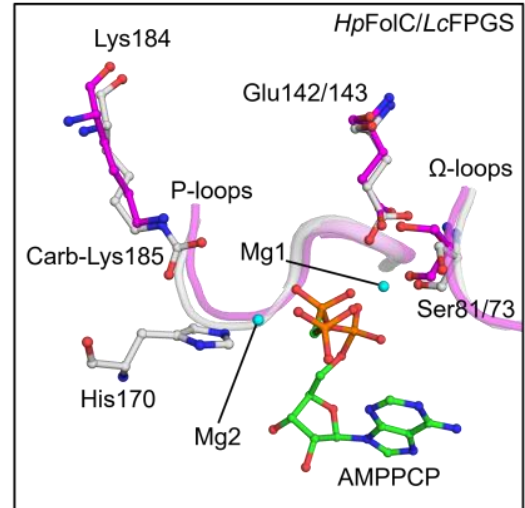

(d)

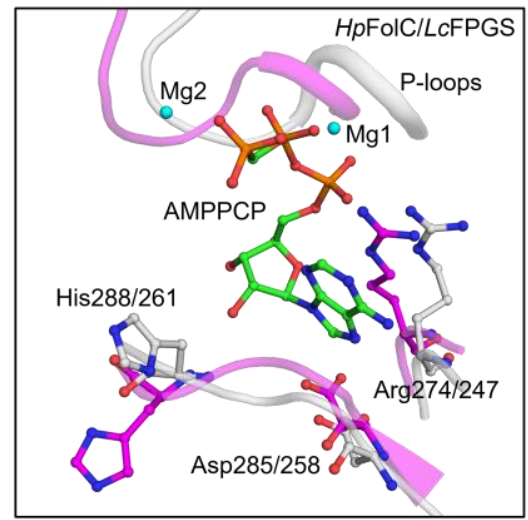

(c)

Figure 4. ATP-binding mode of HpFolC. (a, top left) The structures of apo-HpFolC and L. casei FPGS ( $L c$ FPGS) in complex with an AMPPCP (PDB code: 1JBV) are superposed. HpFolC and LcFPGS are shown in magenta and white cartoon representations, respectively. The AMPPCP molecule is shown with green sticks. Nitrogen, oxygen, phosphorus, and magnesium atoms are colored in blue, red, orange, and cyan, respectively. (b, bottom left) The P-loop residues of LcFPGS and HpFolC are shown with white and magenta sticks, respectively. (c, bottom right) Non-P-loop residues of LcFPGS interacting with the AMPPCP molecule and overlapped $H p F o l C$ residues are shown with white and magenta sticks, respectively. (d, top right) Residues of LcFPGS coordinating Mg1/Mg2 and overlapped $\mathrm{HpFolC}$ residues are shown with white and magenta, respectively.

\subsection{Structural Motifs of HpFolC for ATP Binding via Two Magnesium Ions}

In the ATP binding of FolC, two magnesium ions (Mg1 and $\mathrm{Mg} 2)$ are involved [18-20]. One magnesium (Mg1) is located between $\beta$ - and $\gamma$-phosphates of ATP to interact with them and can be coordinated by the $\Omega$-loop of FolC/FPGS, which is named after its $\Omega$-like shape. The $\Omega$-loop is an additional feature of the $\mathrm{N}$-terminal domains of FolC/FPGSs when compared to other structurally similar nucleotide-binding proteins and is also observable in $\mathrm{HpFolC}$ [19]. The $\Omega$-loop contains approximately 10 residues, in which "Ser-cis-Pro" and "Glu-Arg" motifs are strictly conserved, including their locations. The residue following the Ser-cis-Pro motif is the conserved His/Phe residue, which is discussed below. The next conserved hydrophobic residue (Val84 in HpFolC, Leu101 in MtFolC, Leu86 in EcFolC, and Ile76 in LcFPGS) makes a hydrophobic contact with the C-terminal domain. The $\Omega$-loop holds $\mathrm{Mg} 1$ with the carbonyl group of Ser in the Ser-cis-Pro motif in FolC/FPGS-ATP complex structures [18-20]. Together with the carbonyl group of Ser in the $\Omega$-loop, the side chain of the adjacent conserved Glu (Glu174 of MtFolC, mentioned as Glu176 in the original paper but the numbering in our paper follows the MtFolC sequence in the UniProt database (entry: I6Y0R5); Glu146 of EcFolC; and Glu143 of LcFPGS) 
also coordinates Mg1. The corresponding Ser81 and Glu142 of $H p$ FolC would provide similar functions to other FolC/FPGSs (Figure 4d).

The other magnesium ion (Mg2) coordinates the $\gamma$-phosphate of ATP $[18,20]$. His and carbamylated Lys residues (His173 and Lys188 in EcFolC; His170 and Lys185 in LcFPGS) were observed to hold Mg2 together, and they are also conserved as His169 and Lys184 in HpFolC, respectively (Figure 4d, His169 of $\mathrm{HpFolC}$ is disordered). The carbamylated Lys has been observed in the Mur ligase superfamily [30,31]. However, the carbamylated Lys as well as the coordinated Mg2 was not observed in $\mathrm{HpFolC}$ and $M t$ FolC-AMPPCP complex structures. It seems that Lys carbamylation is essential for the recruitment of $\mathrm{Mg} 2$ and subsequent catalytic reactions. A previous study on $\mathrm{MtFolC}$ suggests that crystallization conditions with a pH higher than 7.0 would result in the carbamylation of the conserved Lys, and further, this carbamylation condition would be a factor for the $\mathrm{pH}$-dependent activity of FolC/FPGS [22]. Although $\mathrm{HpFolC}$ was crystallized at $\mathrm{pH} 7.0$, Lys184 of $\mathrm{HpFolC}$ was not carbamylated.

\subsection{Analyses of Folate Binding of HpFolC, Focused on the $\Omega$-Loop}

As mentioned above, $\mathrm{HpFolC}$ has the conserved His 83 residue preceded by the Ser-cis-Pro motif of the $\Omega$-loop, similar to other FolC/FPGSs (His100 in MtFolC, His85 in EcFolC, and Phe75 in LcFPGS). In the LcFPGS-folate complex structure, Phe75 was shown to interact with the pterin moiety of folate by stacking and a hydrogen bond with its side chain and backbone, respectively. The equivalent His of other FolCs and $\mathrm{HpFolC}$ are also thought to function similarly (Figure 5b). Tyr414 at the opposite side of Phe75 of $L c$ FPGS sandwiches the pterin moiety together with Phe75, and equivalent residues of other FolC/FPGS (Leu380 of $H p$ FolC, Val473 in MtFolC, and His405 in EcFolC) could also provide supporting surfaces against pterin moieties with their side chains (Figure $5 \mathrm{c}$ ). In addition, the glutamate moiety of folate was suggested to extend toward the $\gamma$-phosphate binding site with dynamic movement in the binding mode of folate to LcFPGS [20].

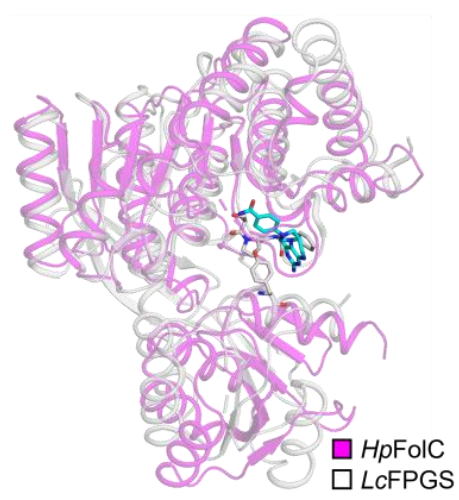

(a)

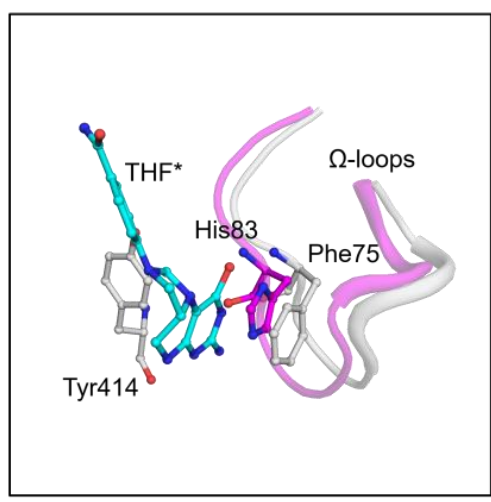

(b)

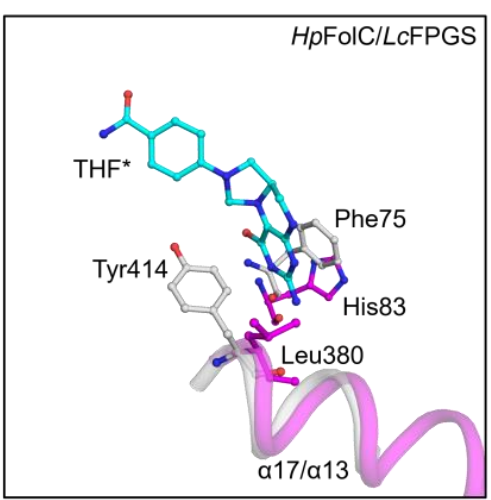

(c)

Figure 5. Folate-binding mode of $H p$ FolC. (a) The structures of apo- $H p F$ FolC and $L c$ FPGS in complex with a tetrahydrofolate (THF) (PDB code: 1JBW) are superposed. HpFolC and LcFPGS are shown in magenta and white cartoon representations, respectively. The residues of LcFPGS interacting with the folate molecule are shown with white sticks, and the folate molecule is shown with cyan sticks. Nitrogen and oxygen atoms are colored in blue and red, respectively. (b) The residues of LcFPGS interacting with the folate molecule and overlapped $\mathrm{H} p \mathrm{FolC}$ residues are shown with white and magenta sticks, respectively. $\Omega$-loops of $H p$ FolC and LcFPGS are shown in the $\mathrm{N}$-terminal domain-superposed view. (c) $\alpha 17$ of $H p F o l C$ and $\alpha 13$ of LcFPGS are shown in the C-terminal domain-superposed view. *The glutamate moiety of THF is disordered in the crystal structure and is omitted.

\subsection{L-Glu Binding Site of FolC/FPGSs}

In the PDB data, the structure of $Y p$ FolC in complex with an $\mathrm{L}-\mathrm{Glu}$ molecule is deposited (PDB code: 3QCZ). In the structure, the loop between the fifth $\beta$-strand and sixth helix (residues Thr167-Tyr179) is open toward the solvent area and the resultant space accommodates the L-Glu molecule. The equivalent 
loop in $H p F o l C$ (residues 163-176) is partially disordered, which implies its flexible movement for ligand binding. However, His169 on this loop (His201 in MtFolC, His173 in EcFolC, and His170 in LcFPGS) was suggested to coordinate Mg2 in the presence of carbamylated Lys, which induces the closed loop conformation. This could provide some clues regarding the mechanism of FolC/FPGS, in which $\mathrm{L}$-Glu binding could occur after dissociation of $\mathrm{Mg} 2$ and $\gamma$-phosphate or $\mathrm{L}$-Glu binding would induce dissociation of $\mathrm{Mg} 2$ and $\gamma$-phosphate.

\section{Conclusions}

Our analyses of the $\mathrm{HpFolC}$ structure revealed that its substrate-recognition components are shared among other reported FolC/FPGS structures. Because the DHFS activity of $H p$ FolC is of great interest for the development of new antibiotics against $H$. pylori, we compared substrate bindings of $H p$ FolC with $L c$ FPGS that would lack DHFS activity as well as human FPGS. However, $L c$ FPGS is structurally similar to bifunctional FolCs, and any outstanding structural difference between FolC and FPGS could not be observed. Thus, further structural and functional investigations of FolC/FPGS are needed for a deeper understanding of the glutamation of folate and DHP in cells. Finally, further structure determination of human FPGS would accelerate the development of selective antibiotics targeting $\mathrm{H} p$ FolC.

Author Contributions: Conceptualization, J.S.P. and B.W.H.; investigation, J.S.P., H.S.K., S.H.P., and M.S.P.; writing—original draft preparation, J.S.P.; writing—review and editing, S.-M.K., H.-J.K., and B.W.H.; supervision, B.W.H.

Funding: This research was supported by the Tumor Microenvironment Global Core Research Center (grant no. 2011-0030001) and the Global Frontier Project (grant no. 2013M3A6A4043695) funded by the National Research Foundation of the Ministry of Science and ICT of Korea. This work was also supported by the Brain Korea (BK21) PLUS program of Seoul National University.

Acknowledgments: We thank the staff at the Pohang Accelerate Laboratory (Pohang, Korea) for the X-ray diffraction experiments. We also thank Se Won Suh for sharing the gene used in the research.

Conflicts of Interest: The authors declare no conflict of interest. The funders had no role in the design of the study; in the collection, analyses, or interpretation of data; in the writing of the manuscript; or in the decision to publish the results.

\section{References}

1. Warren, J.R.; Marshall, B. Unidentified curved bacilli on gastric epithelium in active chronic gastritis. Lancet 1983, 1, 1273-1275. [PubMed]

2. Marshall, B.J.; Warren, J.R. Unidentified curved bacilli in the stomach of patients with gastritis and peptic ulceration. Lancet 1984, 1, 1311-1315. [CrossRef]

3. Harguindey, S.; Pedraz, J.L.; Garcia Canero, R.; Perez de Diego, J.; Cragoe, E.J., Jr. Hydrogen ion-dependent oncogenesis and parallel new avenues to cancer prevention and treatment using a $\mathrm{H}(+)$-mediated unifying approach: pH-related and pH-unrelated mechanisms. Crit. Rev. Oncog. 1995, 6, 1-33. [CrossRef] [PubMed]

4. Malfertheiner, P.; Venerito, M.; Schulz, C. Helicobacter pylori Infection: New Facts in Clinical Management. Curr. Treat. Options Gastroenterol. 2018, 16, 605-615. [CrossRef] [PubMed]

5. Tacconelli, E.; Carrara, E.; Savoldi, A.; Harbarth, S.; Mendelson, M.; Monnet, D.L.; Pulcini, C.; Kahlmeter, G.; Kluytmans, J.; Carmeli, Y.; et al. Discovery, research, and development of new antibiotics: the WHO priority list of antibiotic-resistant bacteria and tuberculosis. Lancet. Infect. Dis. 2018, 18, 318-327. [CrossRef]

6. Shane, B. Folylpolyglutamate synthesis and role in the regulation of one-carbon metabolism. Vitam. Horm. 1989, 45, 263-335. [PubMed]

7. Lu, Y.Z.; Aiello, P.D.; Matthews, R.G. Studies on the polyglutamate specificity of thymidylate synthase from fetal pig liver. Biochemistry 1984, 23, 6870-6876. [CrossRef] [PubMed]

8. Schirch, V.; Strong, W.B. Interaction of folylpolyglutamates with enzymes in one-carbon metabolism. Arch. Biochem. Biophys. 1989, 269, 371-380. [CrossRef] 
9. Lowe, K.E.; Osborne, C.B.; Lin, B.F.; Kim, J.S.; Hsu, J.C.; Shane, B. Regulation of folate and one-carbon metabolism in mammalian cells. II. Effect of folylpoly-gamma-glutamate synthetase substrate specificity and level on folate metabolism and folylpoly-gamma-glutamate specificity of metabolic cycles of one-carbon metabolism. J. Biol. Chem. 1993, 268, 21665-21673.

10. Synold, T.W.; Willits, E.M.; Barredo, J.C. Role of folylpolygutamate synthetase (FPGS) in antifolate chemotherapy; a biochemical and clinical update. Leuk. Lymphoma 1996, 21, 9-15. [CrossRef]

11. Shane, B. Pteroylpoly (gamma-glutamate) synthesis by Corynebacterium species. In vivo synthesis of folates. J. Biol. Chem. 1980, 255, 5649-5654. [PubMed]

12. Bognar, A.L.; Osborne, C.; Shane, B.; Singer, S.C.; Ferone, R. Folylpoly-gamma-glutamate synthetase-dihydrofolate synthetase. Cloning and high expression of the Escherichia coli folC gene and purification and properties of the gene product. J Biol. Chem. 1985, 260, 5625-5630.

13. Fussenegger, M.; Meyer, T.F. Cloning and characterization of the Neisseria gonorrhoeae MS11 folC gene. Mol. Gen. Genet. 1996, 250, 277-285. [PubMed]

14. Sassetti, C.M.; Boyd, D.H.; Rubin, E.J. Genes required for mycobacterial growth defined by high density mutagenesis. Mol. Microbiol. 2003, 48, 77-84. [CrossRef] [PubMed]

15. Wang, P.; Wang, Q.; Yang, Y.; Coward, J.K.; Nzila, A.; Sims, P.F.; Hyde, J.E. Characterisation of the bifunctional dihydrofolate synthase-folylpolyglutamate synthase from Plasmodium falciparum; a potential novel target for antimalarial antifolate inhibition. Mol. Biochem. Parasitol. 2010, 172, 41-51. [CrossRef] [PubMed]

16. Gaudet, P.; Livstone, M.S.; Lewis, S.E.; Thomas, P.D. Phylogenetic-based propagation of functional annotations within the Gene Ontology consortium. Brief. Bioinform. 2011, 12, 449-462. [CrossRef] [PubMed]

17. Bourne, C.R. Utility of the Biosynthetic Folate Pathway for Targets in Antimicrobial Discovery. Antibiotics (Basel) 2014, 3, 1-28. [CrossRef]

18. Mathieu, M.; Debousker, G.; Vincent, S.; Viviani, F.; Bamas-Jacques, N.; Mikol, V. Escherichia coli FolC structure reveals an unexpected dihydrofolate binding site providing an attractive target for anti-microbial therapy. J. Biol. Chem. 2005, 280, 18916-18922. [CrossRef]

19. Sun, X.; Bognar, A.L.; Baker, E.N.; Smith, C.A. Structural homologies with ATP- and folate-binding enzymes in the crystal structure of folylpolyglutamate synthetase. Proc. Natl. Acad. Sci. USA 1998, 95, 6647-6652. [CrossRef]

20. Sun, X.; Cross, J.A.; Bognar, A.L.; Baker, E.N.; Smith, C.A. Folate-binding triggers the activation of folylpolyglutamate synthetase. J. Mol. Biol. 2001, 310, 1067-1078. [CrossRef]

21. Smith, C.A.; Cross, J.A.; Bognar, A.L.; Sun, X. Mutation of Gly51 to serine in the P-loop of Lactobacillus casei folylpolyglutamate synthetase abolishes activity by altering the conformation of two adjacent loops. Acta Crystallogr. D Biol. Crystallogr. 2006, 62, 548-558. [CrossRef] [PubMed]

22. Young, P.G.; Smith, C.A.; Metcalf, P.; Baker, E.N. Structures of Mycobacterium tuberculosis folylpolyglutamate synthase complexed with ADP and AMPPCP. Acta Crystallogr. D Biol. Crystallogr. 2008, D64, 745-753. [CrossRef] [PubMed]

23. Otwinowski, Z.; Minor, W. Processing of X-ray diffraction data collected in oscillation mode. Methods Enzymol. 1997, 276, 307-326. [PubMed]

24. Terwilliger, T.C. SOLVE and RESOLVE: Automated structure solution and density modification. Methods Enzymol. 2003, 374, 22-37. [PubMed]

25. Adams, P.D.; Afonine, P.V.; Bunkoczi, G.; Chen, V.B.; Davis, I.W.; Echols, N.; Headd, J.J.; Hung, L.W.; Kapral, G.J.; Grosse-Kunstleve, R.W.; et al. PHENIX: A comprehensive Python-based system for macromolecular structure solution. Acta Crystallogr. D Biol. Crystallogr. 2010, 66, 213-221. [CrossRef] [PubMed]

26. McCoy, A.J.; Grosse-Kunstleve, R.W.; Adams, P.D.; Winn, M.D.; Storoni, L.C.; Read, R.J. Phaser crystallographic software. J. Appl. Crystallogr. 2007, 40, 658-674. [CrossRef] [PubMed]

27. Emsley, P.; Lohkamp, B.; Scott, W.G.; Cowtan, K. Features and development of Coot. Acta Crystallogr. D Biol. Crystallogr. 2010, 66, 486-501. [CrossRef] [PubMed]

28. Afonine, P.V.; Grosse-Kunstleve, R.W.; Echols, N.; Headd, J.J.; Moriarty, N.W.; Mustyakimov, M.; Terwilliger, T.C.; Urzhumtsev, A.; Zwart, P.H.; Adams, P.D. Towards automated crystallographic structure refinement with phenix.refine. Acta Crystallogr. D Biol. Crystallogr. 2012, 68, 352-367. [CrossRef] [PubMed] 
29. Chen, V.B.; Arendall, W.B., 3rd; Headd, J.J.; Keedy, D.A.; Immormino, R.M.; Kapral, G.J.; Murray, L.W.; Richardson, J.S.; Richardson, D.C. MolProbity: All-atom structure validation for macromolecular crystallography. Acta Crystallogr. D Biol. Crystallogr. 2010, 66, 12-21. [CrossRef] [PubMed]

30. Bertrand, J.A.; Auger, G.; Fanchon, E.; Martin, L.; Blanot, D.; van Heijenoort, J.; Dideberg, O. Crystal structure of UDP-N-acetylmuramoyl-L-alanine:D-glutamate ligase from Escherichia coli. EMBO J. 1997, 16, 3416-3425. [CrossRef]

31. Bertrand, J.A.; Auger, G.; Martin, L.; Fanchon, E.; Blanot, D.; Le Beller, D.; van Heijenoort, J.; Dideberg, O. Determination of the MurD mechanism through crystallographic analysis of enzyme complexes. J. Mol. Biol. 1999, 289, 579-590. [CrossRef] [PubMed]

(C) 2019 by the authors. Licensee MDPI, Basel, Switzerland. This article is an open access article distributed under the terms and conditions of the Creative Commons Attribution (CC BY) license (http://creativecommons.org/licenses/by/4.0/). 